Finance Stochast. 1, 3-24 (1997)

$\overline{\text { Finance and }}$ Stochastics

(c) Springer-Verlag 1997

\title{
On a general class of one-factor models for the term structure of interest rates $^{\star}$
}

\author{
W.M. Schmidt \\ Deutsche Morgan Grenfell, Global Markets, OTC-Derivate, D-60325 Frankfurt am Main, Germany \\ (e-mail: 100347.1533@compuserve.com)
}

\begin{abstract}
We propose a general one-factor model for the term structure of interest rates which based upon a model for the short rate. The dynamics of the short rate is described by an appropriate function of a time-changed Wiener process. The model allows for perfect fitting of given term structure of interest rates and volatilities, as well as for mean reversion. Moreover, every type of distribution of the short rate can be achieved, in particular, the distribution can be concentrated on an interval. The model includes several popular models such as the generalized Vasicek (or Hull-White) model, the Black-Derman-Toy, Black-Karasinski model, and others. There is a unified numerical approach to the general model based on a simple lattice approximation which, in particular, can be chosen as a binomial or $N$-nomial lattice with branching probabilities $1 / N$.
\end{abstract}

Key words: Term structure of interest rates, contingent claim pricing, lattice approximation

JEL classification: G13

Mathematics Subject Classification (1991): 90A09, 60H30

\section{Introduction}

Over the last decade, the increasing volume in interest rate related derivative products has caused immense efforts in modeling the stochastic behaviour of interest rates. Due to the fact that for each point in time there is a whole term structure of interest rates, realistic modeling is much more involved than the popular and useful modeling of stock prices by the famous Black and Scholes

\footnotetext{
* The author thanks an anonymous referee for valuable comments Manuscript received: May 1995; final revision received: June 1996
} 
model. From the practitioners point of view, it is important to develop not only realistic but also computationally tractable models. In particular, a realistic model should be consistent with the current term structure of interest rates and bond prices should tend to the face value of the bond as time approaches the maturity of the bond.

A popular class of models of practical relevance is the class of so-called one-factor models. There, the short rate $\left(r_{t}\right)$ is usually given by a stochastic differential equation of Markovian type

$$
d r_{t}=\mu\left(r_{t}, t\right) d t+\sigma\left(r_{t}, t\right) d W_{t}
$$

driven by a Wiener process $W$. Some examples with their specification of $\mu$ and $\sigma$ are:

$\begin{array}{lcc} & \mu\left(r_{t}, t\right) & \sigma\left(r_{t}, t\right) \\ \text { Ho and Lee } & \theta_{t} & \sigma \\ \text { Vasicek (generalized) } & \theta_{t}-a_{t} r_{t} & \sigma_{t} \\ \text { Cox, Ingersoll, and Ross (generalized) } & \theta_{t}-a_{t} r_{t} & \sigma_{t} \sqrt{ } r_{t} \\ \text { Black and Karasinski } & r_{t}\left(\theta_{t}-a_{t} \ln \left(r_{t}\right)\right) & r_{t} \sigma_{t}\end{array}$

Based on the general framework of arbitrage free pricing, the given dynamics of the short rate determines the stochastic behaviour of zero bond prices and thus, of the whole yield curve.

To be more precise, let us introduce some basic notation. Let $(\Omega, \mathscr{F})$ be a measurable space equipped with a filtration $\mathbf{F}=\left(\mathscr{T}_{t}\right)_{t \geq 0}$. The set $\Omega$ is representing the set of all states of the world and $\mathscr{F}$ (resp. $\mathscr{T}$ ) are the events in the economy observable (resp. until $t$ ). Now let $B(t, M)(\omega), t \leq M, \omega \in \Omega$, denote the price at time $t$ of a zero bond with maturity $M$ and face value of 1 . We assume that $B(., M)$ is an F-adapted stochastic process. The yield curve $\left(r_{t, M}\right)_{M>t}$ is then given by

$$
r_{t, M}=-\frac{\ln B(t, M)}{M-t} .
$$

Supposing the limit

$$
r_{t}=\lim _{M \downarrow t} r_{t, M}
$$

exists, we call $\left(r_{t}\right)_{t \geq 0}$ the short rate process. We denote by

$$
\beta_{t}=\exp \left(\int_{0}^{t} r_{u} d u\right)^{1}
$$

the savings account, which is the price at time $t$ of one unit invested at time 0 and rolled over continuously at the short rate.

Following the spirit of Harrison and Pliska [10], we assume that the market is complete and without arbitrage opportunities. This is essentially equivalent to the following assumption:

${ }^{1}$ We assume that the integral exists. 
There exists a probability $\mathbf{Q}$ on $(\Omega, \mathscr{F})$ such that for every security $S$ without intermediate payments on a time interval $[0, M]$ its price process under the savings account as numeraire, $S_{t} / \beta_{t}$, is a $\mathbf{Q}$-martingale.

This yields

$$
S_{t}=\mathbf{E}_{\mathbf{Q}}\left(\mathrm{e}^{-\int_{t}^{M} r_{u} d u} S_{M} \mid \mathscr{F}_{t}\right)
$$

and, in particular,

$$
B(0, M)=\mathbf{E}_{\mathbf{Q}}\left(\mathrm{e}^{-\int_{0}^{M} r_{u} d u}\right),
$$

which is the condition of consistency with the current term structure of interest rates. For an interest rate dependent contingent claim $C_{M}=C\left(\left(r_{u}\right)_{u \leq M}\right)$ at time $M$, its price at time $t<M$ is then given by

$$
C_{t}=\mathbf{E}_{\mathbf{Q}}\left(\mathrm{e}^{-\int_{t}^{M} r_{u} d u} C_{M} \mid \mathscr{F}_{t}\right)
$$

There are principally four techniques to calculate $C_{t}$ (closely related to each other):

(i) PDE-technique. Using the Markov property of $\left(r_{t}\right)$, the conditional expectation $C_{t}=\mathbf{E}_{\mathbf{Q}}\left(\mathrm{e}^{-\int_{t}^{M} r_{u} d u} C_{M} \mid r_{t}\right)$ is a certain function $C_{t}=C\left(t, M, r_{t}\right)$ which, by the famous Feynman-Kac theorem (cf. [18]), turns out to be a solution of a partial differential equation (PDE). Except some particular cases (e.g. the generalized Vasicek or Cox-Ingersoll-Ross model), the PDE can not be solved explicitly. However, numerical methods are available.

(ii) Numeraire change. This technique is basically a trick for easier calculation of the expectation (1) by choosing an appropriate numeraire in which all securities are measured. For example, replacing the savings account $\beta_{t}$ by the zero bond $B(t, M)$ as new numeraire and the measure $\mathbf{Q}$ on $\mathscr{T}_{T}$ by $\mathbf{Q}_{M}$ with

$$
d \mathbf{Q}_{M}=\frac{\mathrm{e}^{-\int_{0}^{M} r_{u} d u}}{B(0, M)} d \mathbf{Q}
$$

the price $C_{0}$ of the contingent claim $C$ is

$$
C_{0}=B(0, M) \mathbf{E}_{\mathbf{Q}_{M}} C \text {. }
$$

Knowing the distribution of $C$ under the new probability $\mathbf{Q}_{M}$, the expectation can be calculated. For interest rate contingent claims, this technique was introduced in [4]. The use of this powerful technique is demonstrated e.g. in [5], [6], [7].

(iii) Weak convergence. Suppose we have a sequence $r^{n}$ of stochastic processes weakly converging to $r: r^{n} \Rightarrow r$. Then under some technical conditions,

$$
\mathbf{E}_{\mathbf{Q}}\left(\mathrm{e}^{-\int_{0}^{M} r_{u}^{n} d u} C\left(r^{n}\right)\right) \rightarrow \mathbf{E}_{\mathbf{Q}}\left(\mathrm{e}^{-\int_{0}^{M} r_{u} d u} C(r)\right)
$$


Suppose now $r^{n}$ is a Markov process with a discrete state space. Then the expectation $\mathbf{E}\left(\mathrm{e}^{-\int_{0}^{M} r_{u}^{n} d u} C\left(r^{n}\right)\right)$ can be calculated by so-called backward induction, which is based on the rule of iterated conditional expectations and the Markov property. This is the core of the popular lattice methods (cf. Hull and White [12],[13],[14], [15]) which are also related to explicit finite difference methods in the PDE-approach.

(iv) Monte-Carlo simulation. The expectation $\mathbf{E}\left(\mathrm{e}^{-\int_{0}^{M} r_{u} d u} C(r)\right)$ is approximated by simulating a large number of realizations of $\mathrm{e}^{-\int_{0}^{M} r_{u} d u} C(r)$ and calculating its arithmetic mean, which, by the law of large numbers, tends to the expectation. This technique is very time consuming since a large number of simulations is necessary to obtain sufficiently precise results. Improvements can be achieved using several variance reduction techniques.

The present paper introduces a general class of one-factor models and a socalled lattice approximation of the short rate which is then used in the framework of approach (iii) to evaluate prices of interest rate dependent contingent claims. Also, we discuss the calibration of our model to the current term structure of interest rates and to other market data.

\section{A general class of term structure models}

In this section, we are going to propose a general class of one-factor term structure models. The model allows for a perfect fitting of a given term structure of interest rates and volatilities as well as for mean reversion. One of the major advantages of our approach is, that every type of distribution of the short rate can be achieved.

\subsection{The model}

Rather than setting up a model implicitly by a stochastic differential equation for the short rate, our approach is based on an explicit equation for the short rate. We will consider the following model for the short rate $\left(r_{t}\right)$ :

$$
r_{t}=F\left(f(t)+g(t) W_{T(t)}\right), \quad t \geq 0,
$$

where $W$ is a Wiener process on a filtered probability space $(\Omega, \mathscr{F}, \mathbf{Q}), \mathbf{F}^{W}$, and

$$
f \mid[0, \infty) \longrightarrow \mathbf{R},
$$

is a continuous function, 


$$
g \mid[0, \infty) \longrightarrow(0, \infty)
$$

is continuous and positive, and,

$$
\begin{gathered}
T \mid[0, \infty) \longrightarrow[0, \infty), T(0)=0, \\
F \mid \mathbf{R} \longrightarrow \mathbf{R},
\end{gathered}
$$

are both continuous strictly increasing functions. The process $W_{T(t)}$ is a so-called time-changed Wiener process; it is a Wiener process running with a new (deterministic) clock $T(t)$. The information generated by observing the short rate is given by the filtration $\mathbf{F}=\left(\mathscr{T}_{t}\right)$, where $\mathscr{T}_{t}=\mathscr{F}_{T(t)}^{W}$.

As we shall see in the next section, the generalized Vasicek and the BlackDerman-Toy, or Black-Karasinski, model are special cases of our general model.

Let us now discuss the role of the ingredients of the model. The random variable $X_{t}=f(t)+g(t) W_{T(t)}$ is Gaussian with mean $f(t)$ and variance $g^{2}(t) T(t)$. The correlation of $X_{t}$ and $X_{s}$ is $\begin{gathered}T(t \wedge s) \\ \sqrt{ } T(t) T(s)\end{gathered}$. Thus the time-change $T(t)$ determines primarily the correlation structure of the process $X_{t}$.

The function $F$ transforms the Gaussian variable $X_{t}$ into a random variable with distribution function $N \circ F^{(-1) 2}$. Choosing a suitable function $F$, we are able to realize every type of continuous distribution of the short rate. In particular, if the image $F(\mathbf{R})$ is an interval $F(\mathbf{R})=\left(r_{*}, r^{*}\right)$, the short rate $r_{t}$ takes values in $\left(r_{*}, r^{*}\right)$ only, never reaching the upper and lower boundaries $r_{*}, r^{*}$.

The function $f(t)$ is used for the fitting of the current term structure of interest rates; in particular, $f(0)=F^{(-1)}\left(r_{0}\right)$.

Since the variance of $X_{t}$ is just $g^{2}(t) T(t)$, the function $g(t)$ together with $T(t)$ determine the variance of the short rate.

The function $g(t)$ can be interpreted as a (positive or negative) mean reversion force. For example, let $g(t)=\mathrm{e}^{-a t}$ with some positive $a$. Then $g(t)$ causes a reduction of the stochastic term in the definition of $r_{t}$, and this reduction increases as $t$ increases. If, moreover, $T(t)=\int_{0}^{t} g^{-2}(u) \sigma^{2} d u$ as in the simple Vasicek, or Black-Karasinski, model, the variance of $F^{(-1)}\left(r_{t}\right)=f(t)+g(t) W_{T(t)}$ will tend to the constant level $\sigma^{2} / 2 a$ if $t$ increases to infinity. If $g^{2}(t)$ decreases even faster than $T(t)$, i.e. $g^{2}(t) T(t) \rightarrow 0$, we have $\left|r_{t}-F(f(t))\right| \rightarrow 0$.

Following the general theory (cf. Sect. 1) we assume that:

For every security $S$ without intermediate payments on a time interval $[0, T]$ its price process under the savings account as numeraire, $S_{t} / \beta_{t}$, is a $\mathbf{Q}$-martingale.

Remarks. 1. A slightly more general model would be

$$
r_{t}=F\left(t, W_{T(t)}\right), t \geq 0,
$$

and our numerical approach below would also work for this model. Nevertheless, the additional structure in our setup (2) will give us more insight into the role of

\footnotetext{
${ }^{2} N$ denotes the Gaussian cummulative distribution function and $F^{(-1)}$ is the inverse function to $F$.
} 
the ingredients of the model, and it will simplify the calibration of the model to market data.

2. Observe that our general model (2) is a generalization of a class of so-called Brownian-path independent models introduced by Jamshidian [17]. The main difference to these models is the time-change in the Wiener process which allows us to cover the majority of the popular models used in practice.

\subsection{Examples}

Let us now review how several popular models fit into our general model.

(i). The generalized Vasicek model. In this model, the short rate $\left(r_{t}\right)$ is given by the stochastic differential equation

$$
d r_{t}=\left(\theta_{t}-a_{t} r_{t}\right) d t+\sigma_{t} d B_{t},
$$

with some Wiener process $\left(B_{t}\right)$. The solution of this linear stochastic differential equation is known to be given by (cf. e.g. [18])

$$
r_{t}=g(t)\left[r_{0}+\int_{0}^{t} g^{-1}(u) \theta_{u} d u+\int_{0}^{t} g^{-1}(u) \sigma_{u} d B_{u}\right],
$$

where $g(t)=\mathrm{e}^{-\int_{0}^{t} a_{u} d u}$. The source of randomness in $r_{t}$ is a stochastic Ito integral w.r.t. the Wiener process $B$ and with a deterministic integrand. We now use a well-known lemma (cf. e.g. [18], Ch.3.4)

Lemma 1. Let $h \mid[0, \infty) \rightarrow \mathbf{R}$ be such that $\int_{0}^{t} h^{2}(u) d u<\infty, t \geq 0$, and consider the stochastic integral $\int_{0}^{t} h(u) d B_{u}$. Then there exists a Wiener process $W$ such that

$$
\int_{0}^{t} h(u) d B_{u}=W_{T(t)}, t \geq 0
$$

where $T \mid[0, \infty) \rightarrow[0, \infty)$ is given by

$$
T(t)=\int_{0}^{t} h^{2}(u) d u .
$$

Applying this lemma, the short rate process in the generalized Vasicek model can by written as

$$
r_{t}=f(t)+g(t) W_{T(t)}, t \geq 0,
$$

where

$$
\begin{aligned}
g(t) & =\mathrm{e}^{-\int_{0}^{t} a_{u} d u} \\
f(t) & =g(t) r_{0}+g(t) \int_{0}^{t} g^{-1}(u) \theta_{u} d u \\
T(t) & =\int_{0}^{t} g^{-2}(u) \sigma_{u}^{2} d u .
\end{aligned}
$$


Consequently, the generalized Vasicek model fits into our general model for the case $F(x)=x$.

(ii). The Black-Derman-Toy and the Black-Karasinski model. In the BlackKarasinski model, the short rate $\left(r_{t}\right)$ is given by the stochastic differential equation

$$
d \ln \left(r_{t}\right)=\left(\theta_{t}-a_{t} \ln \left(r_{t}\right)\right) d t+\sigma_{t} d B_{t},
$$

with some Wiener process $\left(B_{t}\right)$. The solution of this equation is

$$
\ln \left(r_{t}\right)=g(t)\left[\ln \left(r_{0}\right)+\int_{0}^{t} g^{-1}(u) \theta_{u} d u+\int_{0}^{t} g^{-1}(u) \sigma_{u} d B_{u}\right]
$$

where $g(t)=\mathrm{e}^{-\int_{0}^{t} a_{u} d u}$. Again, using Lemma 1, we see that the short rate process satisfies

$$
r_{t}=\mathrm{e}^{f(t)+g(t) W_{T(t)}}, t \geq 0
$$

where

$$
\begin{aligned}
g(t) & =\mathrm{e}^{-\int_{0}^{t} a_{u} d u} \\
f(t) & =g(t) \ln \left(r_{0}\right)+g(t) \int_{0}^{t} g^{-1}(u) \theta_{u} d u \\
T(t) & =\int_{0}^{t} g^{-2}(u) \sigma_{u}^{2} d u
\end{aligned}
$$

In case of the continuous limit of the popular Black-Derman-Toy model, we have $a_{t}=-\sigma_{t}^{\prime} / \sigma_{t},{ }^{3}$ which implies $g(t)=\sigma_{t} / \sigma_{0}$ and $T(t)=t \sigma_{0}^{2}$. Therefore, in the Black-Derman-Toy model the short rate process $\left(r_{t}\right)$ reduces to

$$
r_{t}=\mathrm{e}^{f(t)+\sigma(t) W_{t}}, t \geq 0
$$

Both models fit into our general model for the case $F(x)=\mathrm{e}^{x}$.

(iii). The quadratic Gaussian model. In the quadratic Gaussian model, the short rate $\left(r_{t}\right)$ is a second order polynomial of a certain state variable which is Gaussian (cf. e.g. [6]). For example, let

$$
d \xi_{t}=\left(a_{1}(t)+a_{2}(t) \xi_{t}\right) d t+b(t) d B_{t}
$$

and define $r_{t}=\xi_{t}^{2}$. As we have seen in our first example, $\xi_{t}$ is of the form $\xi_{t}=f(t)+g(t) W_{T(t)}$. Thus $r_{t}=F\left(f(t)+g(t) W_{T(t)}\right)$ with $F(x)=x^{2}$. In particular, let $a_{1}(t) \equiv 0,2 a_{2}(t)=-a_{t}$ and $2 b(t)=\sigma_{t}$. Then by Ito's formula

$$
d r_{t}=\left(b^{2}(t)-a_{t} r_{t}\right) d t+\sigma_{t} \sqrt{ } r_{t} d \tilde{B}_{t}
$$

where $\tilde{B}$ is a certain Wiener process. But this is a special case of the well-known Cox-Ingersoll-Ross model with time dependent coefficients.

${ }^{3} \sigma_{t}^{\prime}$ denotes the derivative of $\sigma_{t}$. It is assumed that $\sigma$ is smooth. 
(iv). The Sandmann-Sondermann model. Sandmann and Sondermann [19] propose a model which dynamically combines properties of a lognormal and a normal model. Their approach is to model the effective annual rate by a lognormal setup and then transform this into a model for the continuously compounded rate. One motivation for this approach was, that lognormal models for the continuously compounded rate yield infinite expected rollover returns, whereas the model proposed by Sandmann and Sondermann overcomes this drawback. The continuous limit of their model is given by

$$
\begin{aligned}
r_{t} & =\ln \left(1+\xi_{t}\right), \text { where } \\
d \xi_{t} & =\mu(t) \xi_{t} d t+\sigma(t) \xi_{t} d B_{t},
\end{aligned}
$$

with some Wiener process $B$. The solution $\left(\xi_{t}\right)$ of the second equation is given by

$$
\begin{aligned}
\xi_{t} & =\exp \left(\ln \left(\xi_{0}\right)+\int_{0}^{t} \sigma(u) d B_{u}+\int_{0}^{t}\left(\mu(u)-\frac{1}{2} \sigma^{2}(u)\right) d u\right) \\
& =\exp \left(W_{T(t)}+f(t)\right)
\end{aligned}
$$

where

$$
T(t)=\int_{0}^{t} \sigma^{2}(u) d u, f(t)=\ln \left(\xi_{0}\right)+\int_{0}^{t}\left(\mu(u)-\frac{1}{2} \sigma^{2}(u)\right) d u
$$

and $W$ is a Wiener process. Consequently, the short rate in the SandmannSondermann model is given by

$$
r_{t}=F\left(f(t)+W_{T(t)}\right) \text {, with } F(x)=\ln \left(1+\mathrm{e}^{x}\right) .
$$

The model in its original form does not incorporate mean reversion. However, we can easily modify the model to involve mean reversion. To this end let, for example, $g(t)=\mathrm{e}^{-\int_{0}^{t} a(s) d s}$ with some deterministic integrable function $a(s)$. Then a generalization of the Sandmann-Sondermann model which includes mean reversion would be

$$
r_{t}=F\left(f(t)+g(t) W_{T(t)}\right), \text { with } F(x)=\ln \left(1+\mathrm{e}^{x}\right) .
$$

\section{A unified lattice approach}

The reason for introducing our model (2) was not only its generality, but also to present a simple numerical approach to the valuation of interest rate dependent claims, based on a lattice technique as explained in (iii) of the introduction. The main task is to find an appropriate sequence $r^{n}$ of processes (weakly) converging to $r$ and such that expressions of the form

$$
\mathbf{E}\left(\mathrm{e}^{-\int_{0}^{T} r_{u}^{n} d u} C\left(r^{n}\right)\right)
$$

can be calculated more easily. 
Hull and White (cf. [14], [15]) propose an approach based upon a trinomial lattice approximation of $r$. Due to the fact that $r$ is an inhomogeneous diffusion, the probabilities of the branchings in their lattice depend on the specific point in time and on the state of $r^{n}$. Therefore, building the lattice on a computer requires considerable memory resources.

Our approach is much simpler; we will provide a method to construct a simple and easy to implement lattice approximation for $r$. As a special case we obtain a $N$-nomial lattice, where the probabilities for all branchings are equal and $1 / N$.

\subsection{Constructing a lattice for the short rate}

Since the short rate $\left(r_{t}\right)$ is a deterministic (yet time dependent) function of a timechanged Wiener process $\left(W_{T(t)}\right)$, it suffices to find a suitable lattice approximation for $\left(W_{T(t)}\right)$. This is easily accomplished using a generalization of the well-known Donsker's invariance principle (cf. [8]).

Lemma 2. For every $n$ let $0=t_{0}^{n}<t_{1}^{n}<\ldots$ be a discretization of the time axis satisfying $\sup _{i}\left|t_{i}^{n}-t_{i-1}^{n}\right| \downarrow 0$ as $n \rightarrow \infty$. Given a sequence $\Delta_{n} \downarrow 0$, for every $n$ let $\left(Y_{i}^{n}\right)_{i=1,2, \ldots}$ be a sequence of independent random variables taking values in the set $L_{n}=\left\{k \Delta_{n}: k=0, \pm 1, \ldots, \pm K\right\}$. Denote by $p_{i}^{n}(k)$ the probability of taking the value $k \Delta_{n}: p_{i}^{n}(k)=\mathbf{Q}\left(Y_{i}^{n}=k \Delta_{n}\right)$. Now assume that the probabilities $p_{i}^{n}(k)$ are such that $\mathbf{E}_{\mathbf{Q}} Y_{i}^{n}=0, \operatorname{Var}_{\mathbf{Q}} Y_{i}^{n}=T\left(t_{i}^{n}\right)-T\left(t_{i-1}^{n}\right)$. Define

$$
k^{n}(t)=\sup \left\{i: t_{i}^{n} \leq t\right\}
$$

and

$$
M_{t}^{n}=\sum_{i=1}^{k^{n}(t)} Y_{i}^{n} .
$$

Then the process $\left(M_{t}^{n}\right)$ (weakly) converges to $\left(W_{T(t)}\right)$.

The proof of this lemma is easily accomplished using Theorem 7.1.4 of [8]. For every $n$, the process $\left(M_{t}^{n}\right)$ is a square integrable martingale with jumps bounded by $K \Delta_{n}$. The previsible increasing process in the Doob-Meyer decomposition of the submartingale $\left(M^{n}\right)^{2}$ is just

$$
A_{t}^{n}=\sum_{i=1}^{k^{n}(t)} \operatorname{Var}_{\mathbf{Q}} Y_{i}^{n}=T\left(t_{k^{n}(t)}^{n}\right) .
$$

Consequently, for every $t, A_{t}^{n} \rightarrow T(t)$ as $n \rightarrow \infty$. Moreover, for $n \rightarrow \infty$ the jumps of $M^{n}$ and $A^{n}$ tend to zero uniformly on every compact time interval. Now the assertion of the lemma follows from Theorem 7.1.4 of [8].

Let us illustrate the assertion of the lemma by two simple examples.

Example 1: Binomial lattice with equal branching probabilities of $1 / 2$.

Let $n>0$ and define 


$$
t_{i}^{n}=\inf \{t \geq 0: T(t)>i / n\}, i=0,1,2, \ldots
$$

Let $\left(Y_{i}^{n}\right)_{i=1,2, \ldots}$ be a sequence of independent random variables with $\mathbf{Q}\left(Y_{i}^{n}=\right.$ $\left.\frac{1}{\sqrt{ } n}\right)=\mathbf{Q}\left(Y_{i}^{n}=-\frac{1}{\sqrt{ } n}\right)=\frac{1}{2}$. Then the process

$$
M_{t}^{n}=\sum_{i=1}^{k^{n}(t)} Y_{i}^{n}=\sum_{i=1}^{[n T(t)]} Y_{i}^{n} 4
$$

converges weakly to $\left(W_{T(t)}\right)$. Starting at $M_{0}^{n}=0$ the process $M^{n}$ jumps with equal probability ${ }_{2}^{1}$ by $\pm \frac{1}{\sqrt{ } n}$ at times $t$ where the new clock $T(t)$ first exceeds a level ${ }_{n}^{i}, i=1, \ldots$. This means that the jump times are equidistant in the new clock $T(t)$ but in general their distances in real time $t$ differ from step to step.

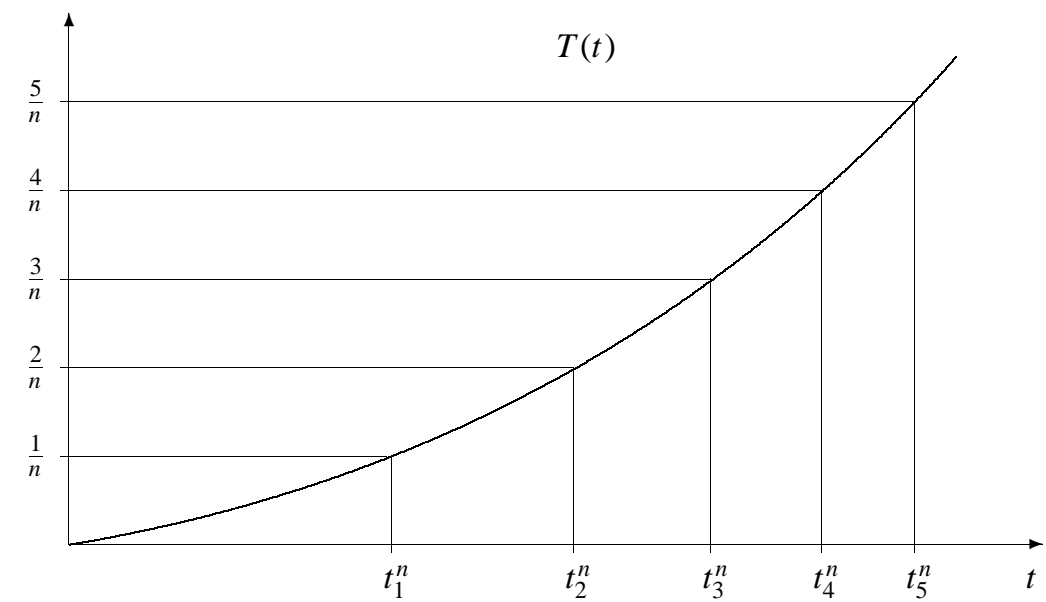

It is easy to generalize the binomial lattice approximation of $\left(W_{T(t)}\right)$ to a $N$-nomial lattice with equal branching probabilities of $1 / N$.

Despite its simplicity, the above binomial lattice approximation has the disadvantage that the discretization in time is completely determined by the timechange $T(t)$. For example, in the simple Vasicek model $T(t)=\frac{\sigma^{2}}{2 a}\left(\mathrm{e}^{2 a t}-1\right), a>0$ and the time-change increases exponentially causing a relatively tight discretization in time for large time points $t$ compared to small time points.

This shortcoming can be avoided using a lattice approximation with more than two branches in every time step, which allows us to chose the discretization in time to a certain extend independently from the time-change $T(t)$. The next example seems to be the simplest example in this direction. It gives the freedom to chose an appropriate sequence of time discretizations. However, in order to match the covariance structure of the process $\left(W_{T(t)}\right)$ in the limit, we have to give up something and make the branching probabilities varying from step to step.

Example 2: Trinomial lattice with time varying branching probabilities. Let $0=t_{0}^{n}<t_{1}^{n}<\ldots$ be a sequence of discretizations of the time axis and let

${ }^{4}[n t]$ denotes the integer part of $n t$. 
$\Delta_{n} \downarrow 0$ be the grid width in the state space of our lattice approximation. We assume that

$$
\frac{T\left(t_{i}^{n}\right)-T\left(t_{i-1}^{n}\right)}{\Delta_{n}^{2}}<1, i=1,2, \ldots
$$

Now for every $n$ let $\left(Y_{i}^{n}\right)_{i=1,2, \ldots}$ be a sequence of independent random variables taking values in $\left\{-\Delta_{n}, 0, \Delta_{n}\right\}$ with probabilities

$$
\begin{gathered}
\mathbf{Q}\left(Y_{i}^{n}=-\Delta_{n}\right)=\mathbf{Q}\left(Y_{i}^{n}=\Delta_{n}\right)=p_{i}^{n}=\frac{T\left(t_{i}^{n}\right)-T\left(t_{i-1}^{n}\right),}{2 \Delta_{n}^{2}}, \\
\mathbf{Q}\left(Y_{i}^{n}=0\right)=1-2 p_{i}^{n} .
\end{gathered}
$$

Then the process $\left(M_{t}^{n}\right)$ defined by (3) converges weakly to $\left(W_{T(t)}\right)$.

Remark. Assuming condition (4) is fulfilled there is also a binomial lattice approximation with time varying branching probabilities but now with time varying jumps. For every $n$ let $Y_{i}^{n}$ take the two values $\delta_{i}^{n}:=\Delta_{n}-$ $\sqrt{ } \Delta_{n}^{2}-\left(T\left(t_{i}^{n}\right)-T\left(t_{i-1}^{n}\right)\right), \delta_{i}^{n}-2 \Delta_{n}$ with probabilities

$$
\mathbf{Q}\left(Y_{i}^{n}=\delta_{i}^{n}\right)=1-\frac{\delta_{i}^{n}}{2 \Delta_{n}}, \mathbf{Q}\left(Y_{i}^{n}=\delta_{i}^{n}-2 \Delta_{n}\right)=\frac{\delta_{i}^{n}}{2 \Delta_{n}} .
$$

Then $\left(M_{t}^{n}\right)$ given by (3) converges weakly to $\left(W_{T(t)}\right)$. Observe that although the jumps are varying from time step to time step the approximation gives a lattice, i.e. is recombining. Indeed, the nodes of the lattice at time $t_{i}^{n}$ are $\left\{\sum_{j=1}^{i} \delta_{j}^{n}-\right.$ $\left.2 k \Delta_{n}: k=0,1, \ldots, i\right\}$.

Based upon Lemma 2 we are now able to give a lattice approximation for the short rate process $\left(r_{t}\right)$ given by (2),

$$
r_{t}=F\left(f(t)+g(t) W_{T(t)}\right)
$$

From the above approximation for $\left(W_{T(t)}\right)$ and from the continuity of the functions $F, f, g$, we get the following simple approximating lattice for $\left(r_{t}\right)$.

Let $0=t_{0}^{n}<t_{1}^{n}<\ldots, \Delta_{n}$ and the sequence of random variables $\left(Y_{i}^{n}\right)_{i=1,2, \ldots}$ be as in Lemma 2. The approximating lattice $r^{n}$ at $t_{i}^{n}$ takes the values

$$
r_{t_{i}^{n}}^{n}(j)=F\left(f\left(t_{i}^{n}\right)+g\left(t_{i}^{n}\right) j \Delta_{n}\right), j=-i K,-i K+1, \ldots, i K-1, i K,
$$

and, from a node $F\left(f\left(t_{i}^{n}\right)+g\left(t_{i}^{n}\right) j \Delta_{n}\right)$ at time $t_{i}^{n}$, it branches with probability $p_{i+1}^{n}(k)$ to

$$
r_{t_{i+1}^{n}}^{n}(j+k)=F\left(f\left(t_{i+1}^{n}\right)+g\left(t_{i+1}^{n}\right)(j+k) \Delta_{n}\right)
$$

at time $t_{i+1}^{n}$.

In the case of the binomial lattice of Example 1 we have $\Delta_{n}=\frac{1}{\sqrt{ } n}, K=1$, $p_{i}^{n}(-1)=p_{i}^{n}(1)={ }_{2}^{1}$ and $p_{i}^{n}(0)=0$, and we can illustrate the lattice approximation with the following picture 


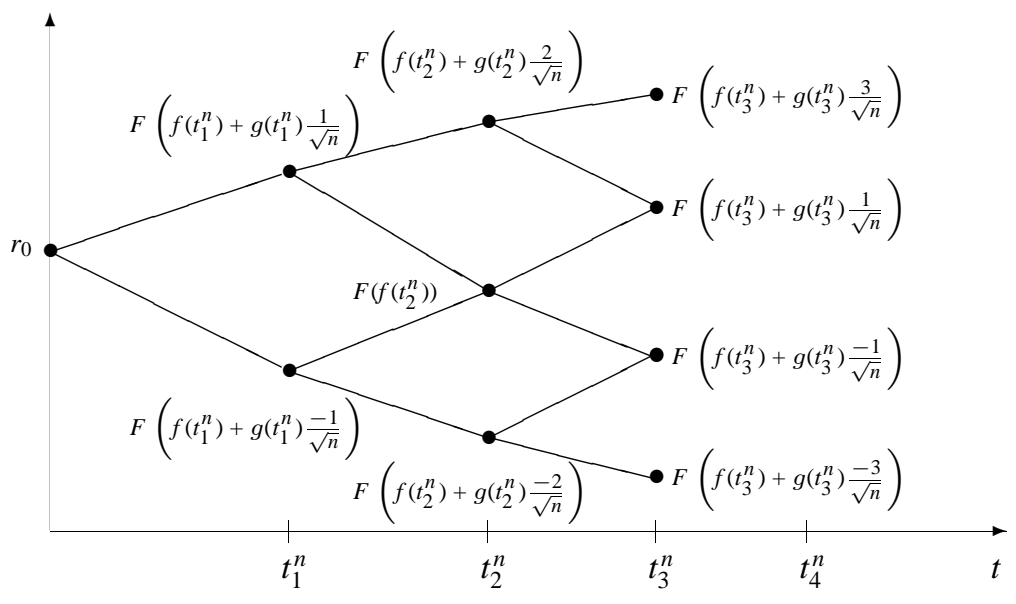

Observe that building the lattice for $\left(r_{t}\right)$ as in Example 1 does not require extra memory on a computer; once the functions $F, f, g$ and the time-change $T(t)$ are known, all nodes of the lattice and their probabilities are known. The same holds true for a trinomial lattice as in Example 2; given the time discretization and lattice width, the approximating lattice $\left(r_{t}^{n}\right)$ is completely determined by $F, f, g$ and the time-change $T(t)$.

Calibrating the model to market data is done by estimating the functions $F, f, g$ and $T(t)$. For example, as explained in Sect. 2.1, the function $f$ determines the term structure of interest rates implied by the model. Therefore, calibrating the model to the current term structure of interest rates, requires estimating the function $f$. We will address to these problems in Sects. 3.2 and 3.3.

Usually, interest rate related contingent claims depend not directly on the short rate but rather on several zero bond prices. Therefore, we are interested in a lattice approximation for prices of zero bonds. Using our lattice for the short rate, it is easy to obtain a respective lattice approximation $B^{n}(t, M)$ for the price of a zero bond $B(t, M)$. For simplicity of notation we keep $n$ fixed and suppress the upper index indicating the dependence on $n$

Let $i_{M}$ be such that $t_{i_{M}}<M \leq t_{i_{M}+1}$. Then the values for $B^{n}\left(t_{i_{M}}, M\right)$ are

$$
B^{n}\left(t_{i_{M}}, M\right)(j)=\exp \left[-F\left(f\left(t_{i_{M}}\right)+g\left(t_{i_{M}}\right) j \Delta\right)\left(M-t_{i_{M}}\right)\right],
$$

$j=-i_{M} K, \ldots, i_{M} K$. For $i<i_{M}$ the nodes for $B^{n}\left(t_{i}, M\right)$ are calculated backwards

$$
B^{n}\left(t_{i}, M\right)(j)=\sum_{k=-K}^{k=K} \mathrm{e}^{-F\left(f\left(t_{i}\right)+g\left(t_{i}\right)(j+k) \Delta\right)\left(t_{i+1}-t_{i}\right)} p_{i+1}(k) B^{n}\left(t_{i+1}, M\right)(j+k)
$$

$j=-i K,-i K+1, \ldots, i K-1, i K$. Having accomplished this construction, we obtain a lattice approximation $B^{n}(t, M)$ of $B(t, M)$; being at a node $B^{n}\left(t_{i}, M\right)(j)$ 
at time $t_{i}$, the lattice $B^{n}$ branches with a probability of $p_{i+1}(k)$ to $B^{n}\left(t_{i+1}, M\right)(j+k)$, $k=-K, \ldots, K$ at time $t_{i+1}$. Now, the well-known backwards iteration technique can be applied to evaluate European or American options on the zero bond $B(t, M)$. Observe, that in general it is necessary to construct the lattice for the zero bond until its maturity and not only until the maturity of the option, which can be very time and memory consuming.

A remarkable exception is provided by Gaussian models for which $F(x)=x$. For these models, prices of zero bonds, at every point in time $t$, can be expressed analytically as a function of the short rate at time $t$. Thus, our lattice for the short rate immediately gives us a lattice for every zero bond. We will investigate this in more detail in Sect. 4.

\subsection{Fitting the model to the term structure of interest rates}

Suppose now we know only the functions $F, g$ and the time-change $T(t)$ of our model. The unknown function $f$ has to be determined in order to calibrate the model to the current term structure of interest rates $B(0, M), 0<M$. We use the known technique of forward induction (cf. [17]).

Suppose we are in our approximating lattice framework with $t_{i}=t_{i}^{n}, r_{t_{i}}(j)=$ $r_{t^{n}}^{n}(j)$ etc. as in the preceding section. Let $A D\left(t_{i}, j\right)^{5}$ denote the price at time 0 of a security that pays one unit at time $t_{i}$ in state $j$, and 0 otherwise. Then, by (1) and the rule of iterated conditional expectations

$$
\begin{aligned}
A D\left(t_{i}, j\right) & =\mathbf{E}_{\mathbf{Q}}\left(\prod_{l=0}^{i-1} \mathrm{e}^{-r_{t_{l}} \cdot\left(t_{l+1}-t_{l}\right)} \mathbf{1}_{\left\{t_{i}, j\right\}}\right) 6 \\
& =\mathbf{E}_{\mathbf{Q}}\left(\mathbf{E}_{\mathbf{Q}}\left(\prod_{l=0}^{i-1} \mathrm{e}^{-r_{t_{l}} \cdot\left(t_{l+1}-t_{l}\right)} \mathbf{1}_{\left\{t_{i}, j\right\}} \mid \mathscr{F}_{t_{i-1}}\right)\right) \\
& =\mathbf{E}_{\mathbf{Q}}\left(\prod_{l=0}^{i-1} \mathrm{e}^{-r_{t_{l}} \cdot\left(t_{l+1}-t_{l}\right)} \mathbf{E}_{\mathbf{Q}}\left(\mathbf{1}_{\left\{t_{i}, j\right\}} \mid \mathscr{F}_{t_{i-1}}\right)\right) .
\end{aligned}
$$

In view of

$$
\mathbf{E}_{\mathbf{Q}}\left(\mathbf{1}_{\left\{t_{i}, j\right\}} \mid \mathscr{T}_{t_{i-1}}\right)=\sum_{k=\max (-K, j-(i-1) K)}^{\min (K, j+(i-1) K)} \mathbf{1}_{\left\{t_{i-1}, j-k\right\}} p_{i}(k)
$$

and, $r_{t_{i-1}} \mathbf{1}_{\left\{t_{i-1}, m\right\}}=r_{t_{i-1}}(m) \mathbf{1}_{\left\{t_{i-1}, m\right\}}$, from the above equality for $A D\left(t_{i}, j\right)$ we obtain

${ }^{5} A D\left(t_{i}, j\right)$ is a so-called Arrow-Debreu price.

${ }^{6} \mathbf{1}_{\left\{t_{i}, j\right\}}$ denotes the random payoff which is one unit in state $j$ at time $t_{i}$, and 0 otherwise. 


$$
\begin{aligned}
& A D\left(t_{i}, j\right)= \\
& \quad \sum_{\substack{\min (K, j+(i-1) K) \\
k=\max (-K, j-(i-1) K)}} A D\left(t_{i-1}, j-k\right) p_{i}(k) \mathrm{e}^{-r_{t_{i-1}}(j-k)\left(t_{i}-t_{i-1}\right)} \\
& j=-i K, \ldots, i K .
\end{aligned}
$$

Moreover, again using (1), we have,

$$
B\left(0, t_{i}\right)=\sum_{j=-(i-1) K}^{(i-1) K} A D\left(t_{i-1}, j\right) \mathrm{e}^{-r_{t_{i-1}}(j)\left(t_{i}-t_{i-1}\right)} .
$$

Equations (5), (6) are the basis for our calibration to the current term structure of interest rates.

First, for $t_{0}=0$ we put $f\left(t_{0}\right)=F^{(-1)}\left(r_{0}\right)$. For $t_{1}$ we have

$$
A D\left(t_{1}, j\right)=\mathrm{e}^{-r_{0} t_{1}} p_{1}(j), j=-K, \ldots, K,
$$

and, using (6),

$$
B\left(0, t_{2}\right)=\mathrm{e}^{-r_{0} t_{1}} \sum_{j=-K}^{K} \mathrm{e}^{-F\left(f\left(t_{1}\right)+g\left(t_{1}\right) j \Delta\right)\left(t_{2}-t_{1}\right)} p_{1}(j) .
$$

The unknown $f\left(t_{1}\right)$ can be calculated from this. More generally, suppose we have already found $f\left(t_{0}\right), \ldots, f\left(t_{i-1}\right)$. Then $A D\left(t_{i}, j\right)$ can be calculated from (5) and by (6)

$$
B\left(0, t_{i+1}\right)=\sum_{j=-i K}^{i K} A D\left(t_{i}, j\right) \mathrm{e}^{-F\left(f\left(t_{i}\right)+g\left(t_{i}\right) j \Delta\right)\left(t_{i+1}-t_{i}\right)} .
$$

From this equation, we calculate $f\left(t_{i}\right)$. Usually, a simple numeric algorithm such as the Newton algorithm or regula falsi works quite well.

\subsection{Calibrating the model to further market data}

As outlined in Sect.2.1, the time-change $T(t)$ and the function $g(t)$ together determine the volatility and the mean reversion of the short rate. There is a compelling need for calibrating these inputs of our model to match further information from the market. From the point of view of a practitioner, observable market information is provided by prices of liquid derivative instruments.

Suppose we have $N$ liquid derivative instruments with prices $P_{1}, \ldots, P_{N}$ and maturities $M_{1}, \ldots, M_{N}$. The prices of these instruments as provided by our model depend upon the choice of $T(t)$ and $g(t)$. We denote them by $C_{1}(T, g), \ldots, C_{N}(T, g)$ always assuming that the model is fitted to the current term structure of interest rates as described in Sect.3.2. Then $T$ and $g$ should be determined such that the difference between $P_{i}$ and $C_{i}(T, g)$ gets as small as possible. One may think of an analogous algorithm as described in Sect. 3.2, 
where we successively determined $f\left(t_{i}\right), i=1,2, \ldots$. However, if for example, $C_{i}(T, g)$ is a price of a swaption, it involves not only the functions $T(t), g(t)$ for $t \leq M_{i}$, but for a much longer period (namely until the end of the swap). It is therefore, in general, unrealistic, to hope for a tractable way to do an analogous successive calibration in a feasible time frame.

One way out of this problem is to describe the functions to be calibrated by a finite set of parameters $\alpha_{1}, \ldots, \alpha_{k}: T(t)=T(t)\left(\alpha_{1}, \ldots, \alpha_{k}\right), g(t)=$ $g\left(t, \alpha_{1}, \ldots, \alpha_{k}\right)$. For example, as in the Vasicek model, $\alpha_{1}=a, \alpha_{2}=\sigma$ and $T(t)={ }_{2 a}^{\sigma^{2}}\left(\mathrm{e}^{2 a t}-1\right), g(t)=\mathrm{e}^{-a t}$. Then one can determine the parameters $\alpha_{1}, \ldots, \alpha_{k}$ by a numerical algorithm such that the deviation of the theoretical prices from the market prices becomes minimal:

$$
\sum_{i=1}^{N}\left(C_{i}(T, g)-P_{i}\right)^{2} \rightarrow \min .
$$

This approach is proposed, for example, by Hull and White [15] and they have found a rather good calibration in the Vasicek model using two parameters.

Other authors use the volatility curve (cf. [2],[3],[17]), to say the current volatility of the yield on zero bonds of all maturities, or prices of contingent claims on the short rate, such as differential caps (cf. [3]) as market information. However, this information is hardly observable from the market. Nevertheless, we describe how our model can be calibrated to such information.

Let $v(t, M), M>t$, denote the volatility structure, that is $v(t, M)$ is the volatility at time $t$ of the yield corresponding to maturity $M$ :

$$
v(t, M)=\operatorname{Var}_{\mathbf{Q}}(\ln (B(t, M))) .
$$

Our aim is to calibrate the model to the term structure of interest rates and, at the same time, to the term structure of volatilities.

Suppose we are in our lattice framework as described in Sect. 3.1. Then the term structure of interest rates is given by $B(0, M), M>0$, and the current volatility structure is

$$
\begin{aligned}
v\left(t_{1}, M\right)= & \sum_{j=-K}^{K} \ln ^{2} B\left(t_{1}, M\right)(j) p_{1}(j) \\
& -\left[\sum_{j=-K}^{K} \ln B\left(t_{1}, M\right)(j) p_{1}(j)\right]^{2}, M>t_{1} .
\end{aligned}
$$

In the binomial lattice approximation of Example 1, the time-change $T(t)$ is determined by the points $t_{i}$ where $T(t)$ first exceeds the number $\frac{i}{n}$. Thus calibrating the time-change $T(t)$ to market data is done by determining successively the points $t_{i}, i=1, \ldots$. 
In the general lattice approximation the calibration of the time-change $T(t)$ is done by solving for the appropriate probabilities $p_{i}(k)$ given the discretization in time $0=t_{0}<t_{1}<\ldots$ However, recall that both, the time-change $T(t)$ and the function $g(t)$ can be chosen in order to calibrate the model to the volatility structure.

To describe the calibration technique by forward induction, we need the prices at time $t_{1}$ and state $m$ of a security that pays one unit at time $t_{i}$ in state $j$, and 0 otherwise, $i \geq 2$. We denote them by $A D_{t_{1}, m}\left(t_{i}, j\right)$. Then using (1) and the rule of iterated conditional expectations, we get the following identities which are analogous to (5) and (6). For $i \geq 2$

$$
\begin{aligned}
A D_{t_{1}, m}\left(t_{i}, j\right)= & \sum_{\substack{k=\max (-K, j-(i-1) K) \\
j \\
j=-i K, \ldots, i K}}^{\min (K, j+(i-1) K)} A D_{t_{1}, m}\left(t_{i-1}, j-k\right) p_{i}(k) \mathrm{e}^{-r_{t_{i-1}}(j-k)\left(t_{i}-t_{i}-1\right)} \\
B\left(t_{1}, t_{i}\right)(m)= & \sum_{j=-(i-1) K}^{(i-1) K} A D_{t_{1}, m}\left(t_{i-1}, j\right) \mathrm{e}^{-r_{t_{i}-1}(j)\left(t_{i}-t_{i-1}\right)}
\end{aligned}
$$

We start our calibration with $f\left(t_{0}\right)=F^{(-1)}\left(r_{0}\right)$ and $t_{1}$ known. Then from (7)

$$
B\left(0, t_{2}\right)=\mathrm{e}^{-r_{0} t_{1}} \sum_{j=-K}^{K} \mathrm{e}^{-F\left(f\left(t_{1}\right)+g\left(t_{1}\right) j \Delta\right)\left(t_{2}-t_{1}\right)} p_{1}(j) .
$$

Using (10) we get

$$
\begin{aligned}
B\left(t_{1}, t_{2}\right)(m) & =\mathrm{e}^{-F\left(f\left(t_{1}\right)+g\left(t_{1}\right) m \Delta\right)\left(t_{2}-t_{1}\right)} \\
v\left(t_{1}, t_{2}\right)= & \sum_{j=-K}^{K} \ln ^{2} B\left(t_{1}, t_{2}\right)(j) p_{1}(j) \\
& -\left[\sum_{j=-K}^{K} \ln B\left(t_{1}, t_{2}\right)(j) p_{1}(j)\right]^{2} .
\end{aligned}
$$

Substituting (12) into (13), together with (11) we obtain two equations which have to be solved for the unknowns $f\left(t_{1}\right), g\left(t_{1}\right), p_{1}\left(\right.$.) (resp. $t_{2}$ in the case of the binomial lattice of Example 1). If $T(t)$ (resp. $g(t)$ ) is given we have, of course, only two unknowns.

Now suppose we have already found $f\left(t_{1}\right), \ldots, f\left(t_{i-1}\right), g\left(t_{1}\right), \ldots, g\left(t_{i-1}\right)$ and the probabilities $p_{1}(),. \ldots, p_{i-1}($.$) (resp. t_{i}$ in the case of the binomial lattice). Then calculate $A D\left(t_{i}, j\right)$ and $A D_{t_{1}, m}\left(t_{i}, j\right)$ from (5) resp. (9). From this we derive $B\left(0, t_{i+1}\right)$ using (6) and $B\left(t_{1}, t_{i+1}\right)(m)$ using (12). Together with (8) applied for $v\left(t_{1}, t_{i+1}\right)$ this yields two equations for the unknowns $f\left(t_{i}\right), g\left(t_{i}\right)$ and the probabilities $p_{i}\left(\right.$.) (resp. $\left.t_{i+1}\right)$ to be solved. 


\subsection{Calibrating volatility in the Black-Derman-Toy model}

In the particular case of the Black-Derman-Toy model, i.e., in the case of $F(x)=$ $\exp (x)$ in our general model, the model can be explicitly calibrated to the term structure of volatilities. Recall from Sect.2.2(ii), that the time change for the Black-Derman-Toy model is just $T(t)=t \sigma_{0}^{2}$.

Suppose the term structure of volatilities is given by the at the money forward volatilities $\sigma(s, t)$ in a Black model, i.e., to value an at the money caplet with reference period $(s, t)$ in a Black (lognormal rate) model, the market uses $\sigma(s, t)$ as appropriate volatility. For a caplet with reference period $(s, t)$ the Black model postulates a lognormal distribution of the rate $z_{s}$ fixed at time $s$ for the period $(s, t)$ with variance of $\ln z_{s}$

$$
\operatorname{Var} \ln z_{s}=\sigma^{2}(s, t) s .
$$

Taking our lattice approximation with discretization in time $t_{i}, i=1, \ldots$, the random variable $r_{t_{i}}$ is the rate at time $t_{i}$ for the period $\left[t_{i}, t_{i+1}\right)$. Thus $r_{t_{i}}$ corresponds to $z_{t_{i}}$ in the Black model. Moreover, $r_{t_{i}}$ has a distribution that is approximately lognormal with variance of $\ln r_{t_{i}}$

$$
\operatorname{Var}_{\mathbf{Q}} \ln r_{t_{i}}=g^{2}\left(t_{i}\right) T\left(t_{i}\right)=g^{2}\left(t_{i}\right) \sigma_{0}^{2} t_{i} .
$$

This leads us to an explicit calibration of our lattice model to the given term structure of volatilities; simply put $\sigma_{0}=1$ and

$$
g\left(t_{i}\right)=\sigma\left(t_{i}, t_{i+1}\right), i=1,2, \ldots
$$

Some tests have shown that, in case of a Black-Derman-Toy model, this calibration technique yields quite good results. The calibration error for every single caplet of a cap is usually less than $1 \%$.

\section{Special example: Gaussian short rates}

For the case of $F(x)=x$ in our general model, the short rate is a Gaussian and a Markovian process. If moreover, the functions $f, g$ and $T(t)$ are differentiable, the model reduces essentially to the generalized Vasicek model, which was studied in detail by several authors (cf. [4],[5],[6],[7],[12],[15],[16]). For the generalized Vasicek model, the price $B(t, M)$ of a zerobond can be expressed explicitly as a function of the short rate $r_{t}$ at time $t$. As a consequence, the model can be analytically calibrated to the term structure of interest rates and to the term structure of volatilities. Also, once having a lattice approximation for the short rate we get immediately an appropriate lattice approximation for all zero bonds.

In this section, we are going to derive these results for our slightly more general Gaussian model.

Let $r_{t}=f(t)+g(t) W_{T(t)}, t \geq 0$. Then by (1) and the Markov property of the short rate $r_{t}$ 


$$
\begin{aligned}
B(t, M) & =\mathbf{E}_{\mathbf{Q}}\left(\mathrm{e}^{-\int_{t}^{M} r_{u} d u} \mid \mathscr{F}_{t}\right) \\
& =\mathbf{E}_{\mathbf{Q}}\left(\mathrm{e}^{-\int_{t}^{M} r_{u} d u} \mid r_{t}\right) \\
& =G\left(t, M, r_{t}\right)
\end{aligned}
$$

with a certain function $G$. We are interested in an explicit expression for $G$. We have

$$
\int_{t}^{M} r_{u} d u=\int_{t}^{M} f(u) d u+\int_{t}^{M} g(u)\left(W_{T(u)}-W_{T(t)}\right) d u+W_{T(t)} \int_{t}^{M} g(u) d u
$$

where the second integral on the right hand side is independent of $\mathscr{T}_{t}$ and the last term is measurable w.r.t. $\mathscr{F}_{t}$. Moreover,

$$
\int_{t}^{M} g(u)\left(W_{T(u)}-W_{T(t)}\right) d u
$$

is Gaussian with expectation 0 and variance $\int_{t}^{M}\left(\int_{u}^{M} g(v) d v\right)^{2} d T(u)$. This yields

$$
\mathbf{E}_{\mathbf{Q}} \mathrm{e}^{-\int_{t}^{M} g(u)\left(W_{T(u)}-W_{T(t)}\right) d u}=\mathrm{e}^{\frac{1}{2} \int_{t}^{M}\left(\int_{u}^{M} g(v) d v\right)^{2} d T(u)}
$$

and thus

$$
\begin{gathered}
B(t, M)= \\
\exp \left\{-\int_{t}^{M} f(u) d u+\frac{1}{2} \int_{t}^{M}\left(\int_{u}^{M} g(v) d v\right)^{2} d T(u)-\frac{r_{t}-f(t)}{g(t)} \int_{t}^{M} g(u) d u\right\} .
\end{gathered}
$$

Observe that the right hand side of this equality provides just the desired function $G\left(t, M, r_{t}\right)$. For $t=0$, in view of $f(0)=r_{0},(15)$ implies

$$
B(0, M)=\exp \left\{-\int_{0}^{M} f(u) d u+\frac{1}{2} \int_{0}^{M}\left(\int_{u}^{M} g(v) d v\right)^{2} d T(u)\right\} .
$$

On the other hand, (16) leads to the following explicit expression for the function $f(t)$

$$
f(t)=\frac{d}{d t}\left(-\ln (B(0, t))+\frac{1}{2} \int_{0}^{t}\left(\int_{u}^{t} g(v) d v\right)^{2} d T(u)\right),
$$

where we assume that the derivative exists. Equation (17) allows an explicit fitting of the model to the current term structure of interest rates. In the same way, calibration of the model to a given term structure of volatilities can be done explicitly. In fact, using (15) we have 


$$
\begin{aligned}
v(t, M) & =\operatorname{Var}(\ln (B(t, M))) \\
& =\operatorname{Var}\left(-\frac{r_{t}}{g(t)} \int_{t}^{M} g(u) d u\right) \\
& =\left(\int_{t}^{M} g(u) d u\right)^{2} T(t) .
\end{aligned}
$$

The unknown time-change $T(t)$, or the unknown function $g$, or both, can be immediately obtained from the volatility structure.

In the case of the original Vasicek model, that is in the case of $g(t)=$ $\mathrm{e}^{-a t}, T(t)=\int_{0}^{t} g^{-2}(u) \sigma^{2} d u$, the above identities simplify considerably to:

$$
\begin{aligned}
& B(t, M)= \\
& \left.\quad \begin{array}{l}
B(0, M) \\
B(0, t)
\end{array}\right) \exp \left\{\begin{array}{c}
r_{t}-f(t) \\
a
\end{array}\left(\mathrm{e}^{-a(M-t)}-1\right)\right. \\
& \left.+\frac{\sigma^{2}}{4 a^{3}}\left[\left(1-\mathrm{e}^{-2 a(M-t)}\right)\left(1-\mathrm{e}^{-2 a t}\right)-4\left(1-\mathrm{e}^{-a(M-t)}\right)\left(1-\mathrm{e}^{-a t}\right)\right]\right\},
\end{aligned}
$$

and

$$
\begin{aligned}
f(t) & =\frac{d}{d t}(-\ln B(0, t))+\frac{\sigma^{2}}{2 a^{2}}\left(\mathrm{e}^{-a t}-1\right)^{2} \\
v(t, M) & =\frac{\sigma^{2}}{2 a^{3}}\left(\mathrm{e}^{2 a t}-1\right)\left(\mathrm{e}^{-a M}-\mathrm{e}^{-a t}\right)^{2} .
\end{aligned}
$$

\section{Some numerical results}

To illustrate the lattice approximation proposed in Sect.3, in this section we present some numerical results on the speed of the convergence and the ability of the model to calibrate to given market prices of caps and swaptions.

In the case of a Gaussian short rate closed form solutions for the prices of caps and swaptions are available. Therefore this model is best suited to test the speed of the convergence of the lattice approximation. We consider a flat interest rate term structure of $6 \%$, i.e. $B(0, t)=\mathrm{e}^{-0.06 t}$. The time change $T(t)$ and the function $g(t)$ are as at the end of Sect. 4 with mean reversion parameter $a=0.22$ and short rate variance $\sigma=0.018$. Observe that these numbers are in line with numbers usually obtained from calibrating the model to market prices of caps. We use the binomial lattice approximation of Sect.3.1, Example 1 to price a 5year cap on a 6-month rate and compare the results with exact prices from closed form solutions. The time from today to 5 years is divided into $N$ non-equidistant time intervals as described in the binomial lattice approximation of Example 1. In the tables below $n$ denotes the number of time steps in the binomial tree from time 0 to the reset date of the respective caplet. 
Table 1. At the money cap $($ strike $=6 \%)$

\begin{tabular}{c|c|c|c|c|c|c|c|c|}
\multicolumn{2}{c|}{} & \multicolumn{2}{c}{$N=50$} & \multicolumn{2}{c|}{$N=100$} & \multicolumn{2}{c}{$N=200$} \\
\hline caplet & exact & lattice & $n$ & lattice & $n$ & lattice & $n$ \\
\hline 1 & 0.2431 & 0.2563 & 1 & 0.2595 & 3 & 0.2390 & 6 \\
2 & 0.3092 & 0.3222 & 3 & 0.2946 & 6 & 0.3097 & 13 \\
3 & 0.3461 & 0.3528 & 5 & 0.3497 & 11 & 0.3484 & 23 \\
4 & 0.3676 & 0.3614 & 8 & 0.3710 & 17 & 0.3693 & 35 \\
5 & 0.3796 & 0.3788 & 12 & 0.3798 & 24 & 0.3802 & 49 \\
6 & 0.3852 & 0.3904 & 17 & 0.3867 & 34 & 0.3864 & 68 \\
7 & 0.3863 & 0.3885 & 22 & 0.3876 & 45 & 0.3863 & 91 \\
8 & 0.3844 & 0.3874 & 29 & 0.3851 & 59 & 0.3840 & 119 \\
9 & 0.3803 & 0.3831 & 38 & 0.3804 & 77 & 0.3804 & 155 \\
sum & 3.1817 & 3.2209 & & 3.1946 & & 3.1837 & \\
error & & $-1.23 \%$ & & $-0.40 \%$ & & $-0.06 \%$ & \\
& & & & & & & \\
\end{tabular}

Table 2. Out of the money cap (strike $=7 \%)$

\begin{tabular}{c|c|c|c|c|c|c|c|c|}
\multicolumn{2}{c|}{} & \multicolumn{2}{c}{$N=50$} & \multicolumn{2}{c|}{$N=100$} & \multicolumn{2}{c}{$N=200$} \\
\hline caplet & exact & lattice & $n$ & lattice & $n$ & lattice & $n$ \\
\hline 1 & 0.0702 & 0.0197 & 1 & 0.0656 & 3 & 0.0641 & 6 \\
2 & 0.1272 & 0.0979 & 3 & 0.1234 & 6 & 0.1274 & 13 \\
3 & 0.1636 & 0.1445 & 5 & 0.1638 & 11 & 0.1655 & 23 \\
4 & 0.1873 & 0.1919 & 8 & 0.1897 & 17 & 0.1883 & 35 \\
5 & 0.2026 & 0.2071 & 12 & 0.1991 & 24 & 0.2020 & 49 \\
6 & 0.2121 & 0.2147 & 17 & 0.2107 & 34 & 0.2134 & 68 \\
7 & 0.2175 & 0.2187 & 22 & 0.2192 & 45 & 0.2175 & 91 \\
8 & 0.2200 & 0.2229 & 29 & 0.2209 & 59 & 0.2205 & 119 \\
9 & 0.2202 & 0.2193 & 38 & 0.2205 & 77 & 0.2209 & 155 \\
\hline sum & 1.6208 & 1.5367 & & 1.6129 & & 1.6195 & \\
error & & $5.19 \%$ & & $0.49 \%$ & & $0.08 \%$ & \\
& & & & & & &
\end{tabular}

Table 3. In the money cap ( strike $=5 \%$ )

\begin{tabular}{|c|c|c|c|c|c|c|c|c|}
\multicolumn{3}{c|}{} & \multicolumn{2}{c}{$N=50$} & \multicolumn{2}{c|}{$N=100$} & \multicolumn{2}{c|}{$N=200$} \\
\hline caplet & exact & lattice & $n$ & lattice & $n$ & lattice & $n$ \\
\hline 1 & 0.5662 & 0.5200 & 1 & 0.5690 & 3 & 0.5631 & 6 \\
2 & 0.6027 & 0.5910 & 3 & 0.5941 & 6 & 0.6016 & 13 \\
3 & 0.6224 & 0.6200 & 5 & 0.6268 & 11 & 0.6197 & 23 \\
4 & 0.6310 & 0.6331 & 8 & 0.6344 & 17 & 0.6313 & 35 \\
5 & 0.6322 & 0.6300 & 12 & 0.6351 & 24 & 0.6339 & 49 \\
6 & 0.6284 & 0.6341 & 17 & 0.6313 & 34 & 0.6287 & 68 \\
7 & 0.6210 & 0.6240 & 22 & 0.6225 & 45 & 0.6209 & 91 \\
8 & 0.6112 & 0.6134 & 29 & 0.6137 & 59 & 0.6121 & 119 \\
9 & 0.5996 & 0.6038 & 38 & 0.6013 & 77 & 0.6005 & 155 \\
\hline sum & 5.5147 & 5.4693 & & 5.5283 & & 5.5117 & \\
error & & $0.82 \%$ & & $-0.25 \%$ & & $0.05 \%$ & \\
\hline
\end{tabular}

Finally we present some results on the quality of the calibration of the model to given market prices of liquid derivatives. We consider a model with lognormally distributed short rate and use a lattice approximation as proposed in 
Table 4. Calibration to at the money caps

\begin{tabular}{c|c|c|c|c|c|c|c|}
\hline maturity & $1 \mathrm{Y}$ & $2 \mathrm{Y}$ & $3 \mathrm{Y}$ & $4 \mathrm{Y}$ & $5 \mathrm{Y}$ & $7 \mathrm{Y}$ & $10 \mathrm{Y}$ \\
\hline $\begin{array}{c}\text { market price } \\
\text { model }\end{array}$ & 0.1808 & 0.8170 & 1.6675 & 2.5458 & 3.4918 & 4.6119 & 6.9072 \\
\hline & 0.1863 & 0.8085 & 1.6503 & 2.5719 & 3.5038 & 4.5924 & 6.9021 \\
\hline
\end{tabular}

Table 5. Calibration to at the money swaptions

\begin{tabular}{c|c|c|c|c|c|}
\multicolumn{2}{|c|}{ maturity } & $3 \mathrm{M}$ & $6 \mathrm{M}$ & $1 \mathrm{Y}$ & $2 \mathrm{Y}$ \\
\hline \multicolumn{2}{|c|}{ swap term } & & & & \\
\hline \multirow{2}{*}{$2 \mathrm{Y}$} & market & 0.3963 & 0.5510 & 0.7823 & 1.0446 \\
& model & 0.3884 & 0.5591 & 0.7796 & 0.9810 \\
\hline \multirow{2}{*}{$3 \mathrm{Y}$} & market & 0.5476 & 0.7296 & 1.0270 & 1.3472 \\
& model & 0.5444 & 0.7666 & 1.0620 & 1.3276 \\
\hline \multirow{2}{*}{$4 \mathrm{Y}$} & market & 0.6688 & 0.9373 & 1.2546 & 1.5280 \\
\hline \multirow{2}{*}{$5 \mathrm{Y}$} & model & 0.6774 & 0.9557 & 1.2843 & 1.5438 \\
\hline \multirow{2}{*}{$7 \mathrm{Yarket}$} & 0.7914 & 1.0704 & 1.4032 & 1.7414 \\
\hline \multirow{2}{*}{$7 \mathrm{Y}$} & model & 0.7647 & 1.0608 & 1.4192 & 1.7820 \\
& model & 0.9055 & 1.2352 & 1.7054 & 2.0582 \\
& & 0.8860 & 1.2593 & 1.6980 & 2.0822 \\
\hline
\end{tabular}

Example 2 of Sect. 3.1 with suitably parametrized functions $g(t), T(t)$ and appropriately chosen time discretization. The model is calibrated simultaneously to market prices of 7 at the money caps and 20 swaptions. The results show a quite good calibration of the model to the market. Observe that in practice one would probably choose less reference options to calibrate to. All prices and the term structure of interest rates refer to the DEM market as of June 24, 1996.

\section{Conclusion}

There is a general one-factor model for the term structure of interest rates based upon an explicit formula for the dynamics of the short rate. The short rate is modeled as a Wiener process with a deterministic new clock which is then subject to an addition of a drift and a transformation of the state space. Essentially every popular short rate model used in practice turns out to be a particular case of this general approach. Several easy to implement lattice approximations of the short rate process all ow pricing of interest related contingent claims using the wellknown backward induction method. In the simplest case the lattice approximation is a binomial lattice with branching probabilities $1 / 2$ but non-equidistant spacing in time. The model can be calibrated to the given term structure of interest rates as well as to given market prices of liquid derivative products or the volatility structure of zero bonds.

\section{References}

1. F. Black: The pricing of commodity contracts. J. Financial Econ. 3, 167-179 (1976)

2. F. Black, E. Derman, W. Toy: A one-factor model of interest rates and its application to treasury bond options. Financial Analysts J. 33-39 (1990) 
3. F. Black, P. Karasinski: Bond and option pricing when short rates are lognormal. Financial Analysts J. 52-59 (1991)

4. N. El Karoui, J. Rochet: A pricing formula for options on coupon bonds. SEEDS Working Paper 72 (1989)

5. N. El Karoui, C. Lepage, R. Myneni, N. Roseau, R. Viswanathan: The valuation and hedging of contingent claims with Markovian interest rates, 1991

6. N. El Karoui, R. Myneni, R. Viswanathan: Arbitrage pricing and hedging of interest rate claims with state variables: I Theory, 1992

7. N. El Karoui, C. Lepage, R. Myneni, N. Roseau, R. Viswanathan: The pricing and hedging of interest rate claims: Applications, 1991

8. S. N. Ethier, T. G. Kurtz: Markov processes. Characterization and convergence. New York: John Wiley \& Sons 1986

9. J. M. Harrison, D. M. Kreps: Martingales and arbitrage in multiperiod security markets. J. Econ. Theory 20, 381-408 (1979)

10. J. M. Harrison, S. R. Pliska: Martingales and stochastic integrals in the theory of continuous trading. Stoch. Processes Appl. 11, 215-260 (1981)

11. T. Ho, S. Lee: Term structure movements and pricing interest rate contingent claims. J. Finance 41, 1011-1029 (1986)

12. J. Hull, A. White: Pricing interest rate derivative securities. Rev. Financial Studies 3, 573-592 (1990)

13. J. Hull, A. White: Valuing derivative securities using the explicit finite difference method. J. Financial Quantitat. Anal. 25, 87-100 (1990)

14. J. Hull, A. White: One-factor interest rate models and the valuation of interest rate derivative securities. J. Financial Quantitat. Anal. 28, 235-254 (1993)

15. J. Hull, A. White: Numerical procedures for implementing term structure models I, II. J. Derivat. 2, (1) 7-16, (2) 37-48 (1994)

16. F. Jamshidian: An exact bond option pricing formula. J. Finance 44, 205-209 (1989)

17. F. Jamshidian: Forward induction and construction of yield curve diffusion models. J. Fixed Income 1, 62-74 (1991)

18. I. Karatzas, S. E. Shreve: Brownian motion and stochastic calculus. Berlin-Heidelberg-New York: Springer 1988

19. K. Sandmann, D. Sondermann: A term structure model and the pricing of interest rate derivatives. Rev. Futures Markets 12, (2), 391-423 (1993)

20. O. Vasicek: An equilibrium characterization of the term structure. J. Financial Econ. 5, 177-188 (1977) 